\title{
The influence of unfractionated and low-molecular weight heparins on the properties of human umbilical vein endothelial cells (HUVEC)
}

\author{
Katarzyna Gąsowska, Beata Naumnik, Katarzyna Klejna, Michał Myśliwiec
}

Department of Nephrology and Transplantation with Dialysis Unit, Medical University of Białystok, Białystok, Poland

\begin{abstract}
Heparins, as anticoagulants widely used in the prophylaxis and treatment of many conditions connected with hypercoagulability, have a potent effect on the vascular endothelium. Unfractionated Heparin (UFH) is characterized by relatively low biological accessibility, short activity time, binding of numerous proteins, as well as unfavorable influence on endothelium and blood platelets. Low-Molecular Weight Heparins (LMWHs), formed by chemical and enzymatic UFH depolymerizations, show a significantly more favorable impact on endothelium, which was confirmed on the HUVEC cultures study models. The studies on the heparins' modulation of angiogenesis process proved the superiority of LMWHs over UFH. It was connected with a better deactivation of growth factors' receptors (e.g. for VEGF165, FGF-2). Comparing the effects of LMWHs and UFH on haemostatic and antiangiogenic properties of HUVEC, significant differences were found as well. A new effect, engaging these compounds in the pathomechanism of an excessive osteoclastogenesis via osteoprotegerin /RANKL/RANK pathway has been discovered recently.
\end{abstract}

Key words: HUVEC, low molecular weight heparin, osteoprotegerin, RANK/RANKL, unfractionated heparin

\section{Introduction}

Heparin was discovered by two independent researchers: Doyon in 1911 and McLean in 1916 year. Doyon detected an anticoagulant produced in the liver, later called antithrombin. In 1916 year, Mc Lean, conducting studies on canine livers, found a compound stimulating the activity of antithrombin, so called heparin that turned out to be an extremely valuable medication. It was used in the prophylaxis of thromboembolic diseases in patients who underwent operations, orthopedic or gynecological procedures, as well as in hemodialysis and many others.

Wide administration of heparin increased the interest in its systemic activity, especially, in its modulator effect on endothelial cells (EC). However, despite many years of studies, side effects of heparin as well as its influence on the endothelium have not been explained completely. The studies on the endothelial cell culture (HUVEC; human umbilical vein endothe-

Correspondence: B. Naumnik, Dept. of Nephrology and Transplantation, Żurawia Str. 14, 15-540 Białystok, Poland; tel./fax.: (+4885) 7434586, e-mail: bnaumnik@poczta.onet.pl lial cells) seem to be of great importance. They enable a better understanding of the processes taking place during heparin administration.

Heparins, as glycosaminoglycans, form chains built up of repeated disaccharide unites. Each such a unit is formed via binding of glucosamine with glucuronic acid.

Heparins are divided into long-chained, e.g. unfractionated heparin (UFH) and short-chained, e.g. lowmolecular weight heparins (LMWHs), depending on the number of polysaccharide chains. There are numerous differences between these two types of heparins regarding dissimilarities in the mechanism of their activity, pharmacokinetics and complications after therapy.

\section{Comparison of basic features of unfractionat- ed and low-molecular weight heparins}

The anticoagulant activity of heparin includes its binding with antithrombin (AT), due to which a slow-acting compound is transformed into a fast inhibitor of thrombin (factor IIa) and factor Xa. A three-part complex is formed on AT, heparin and inhibited coagula- 


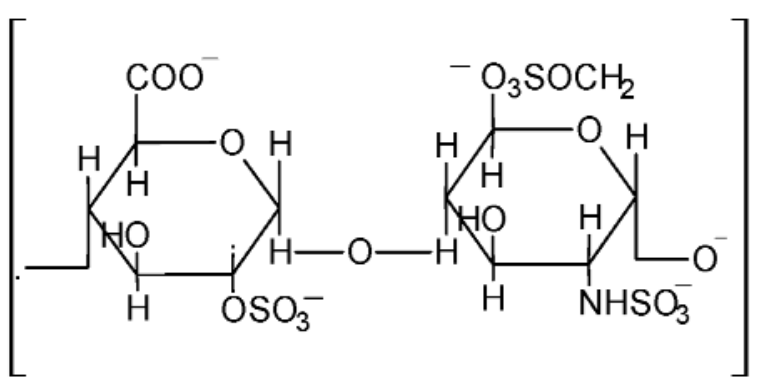

$\mathrm{n}$

Fig. 1. Disaccharide unit repeating itself n-times (depending on the type of heparin) in polysaccharide chains.

tion enzyme. Afterwards, heparin disconnects and can be used again. It inactivates, to a lower degree, other serine proteases such as factors VIIa, IXa, XIa and XII. Another mechanism of heparin activity consists in releasing the inhibitor of an extrinsic coagulation pathway via a tissue factor (TFPI; Tissue Factor Pathway Inhibitor) from the endothelial surface [1]. Polysaccharide chains include pentasaccharide structures that binds heparin with AT by means of the glucosamine radical, intensifying its activity at the same time. The presence of pentasaccharide sequence in the chain enables binding with antithrombin. However, not all molecules show this ability, because chains without pentasaccharide present no anticoagulant activity. Molecules, built up of 18 or more saccharide residues, except for pentasaccharide indispensable to bind AT, possess abilities to inhibit the activity of thrombin. Shorter chains are able to neutralize only factor Xa. At the beginning, heparins with longer chains were synthesized, making it possible, in the comparable degree, to inhibit the activity of factors Xa and IIa. These compounds were called unfractionated heparins of medium molecular weight of $12-15 \mathrm{kDa}$. UFH is obtained from the mucosa of pig's intestines or ox's lungs.

Despite the lack of heparin toxicity, many complications, e.g., hemorrhage or thrombocytopenia may be reported during its application. This fact has caused the research of better anticoagulant agents without side effects. Shortening of polysaccharide chains of heparin via chemical or enzymatic depolymerization and purification has given interesting results. Fractioned heparins, popularly called low-molecular weight heparins, with medium molecular weight of 3.9-6 kDa were obtained in this way [2]. Additionally, these heparins differ with regard to pharmacokinetics, bioaccessibility and many other features (Table 1). Lower binding of LMWHs with plasma proteins than it is observed in case of UFH, plays a major role in their biological accessibility increase. In case of UFH, a medium period of half-life equals 90 minutes, whereas for LMWHs it amounts from 2 to 3 hours after intravenous administration, which entails pharmacokinetic differences as well. LMWHs are also characterized by lower binding with endothelium that was confirmed in a cell culture.
Table 1. Comparison of unfractioned and low molecular heparins (according to Chojnowski 2000, Windyga 2003 modified).

\begin{tabular}{|l|c|c|}
\hline & UFH & LMWH \\
\hline $\begin{array}{l}\text { Mean/medium molecular } \\
\text { weight (kDa) }\end{array}$ & $12-15$ & $3.9-6$ \\
\hline Ratio of anti-Xa/anti-IIa & $1: 1$ & $\begin{array}{c}\text { from } 2: 1 \\
\text { to } 5: 1\end{array}$ \\
\hline Binding with proteins & $\begin{array}{c}\text { HRGP, Fn, Vn, } \\
\text { PF4, vWF }\end{array}$ & Vn \\
\hline Biological accessibility \% & 30 & 90 \\
\hline Half-life period (h) & 1.5 & $2-3$ \\
\hline Binding to endothelium & Yes & No \\
\hline $\begin{array}{l}\text { Increase in vascular wall } \\
\text { permeability }\end{array}$ & Yes & No \\
\hline Influence on platelets & Yes & No \\
\hline
\end{tabular}

Abbreviations: HRGP - glycoprotein rich in histidine , Fn - fibronectin, $\mathrm{Vn}$ - vitronectin, PF4 - platelet factor 4, vWF - von Willebrand factor

Significant profits of LMWHs result from fewer hemorrhages than during UFH administration. This enabled partial elimination of the most serious complication of heparin therapy. A decrease in hemorrhage frequency can be explained by lower affinity of LMWHs to the von Willebrand factor (vWF), which is associated with its normal function in the process of coagulation, mainly consisting in platelet adhesion and factor VIII protection. The next reason for hemorrhage after UFH is an increase in permeability of blood vessels, whereas administration of LMWHs has no such effect. LMWHs, in comparison with UFH, are characterized by less influence on platelet activity which is proved by significantly less frequent thrombocytopenia. Postheparin thrombocytopenia may occur due to two processes. One of them is nonimmunological (HAT; heparin-associated thrombocytopenia), which is the most frequent mild form of thrombocytopenia, without sever clinical symptoms. The other mechanism (HIT; heparin-induced thrombocytopenia), is a severe complication of high mortality. It occurs when antibodies against platelet factor 4 (PF4), mainly from IgG class, form complexes with heparin and activate platelets. Low-molecular weight heparins as medications affecting platelets to a lesser extent, have only partially met expectations, though HIT cases are significantly less frequent after their administration [3].

In the face of suspicion of heparins' different effects on endothelium, only the usage of cell culture techniques has explained and confirmed earlier speculations. The comparison of UFH and LMWHs activity in HUVEC, with special regard to differences in haemostatic, antiangiogenic or anticancerous features, has become of great interest. 


\section{Influence of UFH vs LMWHs on haemostatic factors in HUVEC culture}

Endothelium takes a vital part in the variety of mechanisms such as release of many substances engaged in e.g., pro/anticoagulant balance, angiogenesis as well as inflammatory and immunological processes. Damage to its structure leads to disturbance of multi-organic cooperation that result in numerous pathological changes, such as thrombosis, atherosclerosis and hypertension. There is a layer of glycocalix built up of glycosaminoglycans (GAG), where heparin sulphate predominates. Endothelium, charged negatively by means of glycocalix, pushes away blood morphogenic components [5]. The role of endothelial cells in haemostasis is to inhibit blood coagulation, which is connected with anticoagulant factors. Antithrombin is, undoubtedly, the most important anticoagulant factor of all produced by endothelium, whereas surface GAGs (heparin-like particles) significantly enhance its activity. Exogenous heparin acts like particles forming glycocalix and its administration influences the features of the vascular wall. Endothelial cells release also other substances playing a pivotal role in the maintenance of blood flow, such as TFPI, a tissue type plasminogen activator (t-PA), and thrombomodulin (TM). The activation of TM takes place in the presence of its cofactor - protein S, causing degradation and inactivation of active factors VIII and V. Additionally, protein $\mathrm{C}$ shows profibrinolytic properties via binding of plasminogen activator inhibitor-1 (PAI-1). Heparins can bind vWF and PF4, inhibiting aggregation and activation of platelets. The further studies, confirming and widening former data, were carried out using HUVEC culture. Apart from the anticoagulant activity, there is a procoagulant aspect of heparin's impact on EC - releasing procoagulant factors e.g. tissue factor (TF) or plasminogen activator inhibitor-1 (PAI-1).

The length of polysaccharide chains is of great importance in haemostatic balance. The studies performed on HUVEC culture confirmed the earlier findings regarding the stimulating activity of heparin on TF release [6]. An outstandingly high level of TF antigen, regardless a dose, was observed in the test with UFH. In case of LMWHs, the highest increase was observed after 24-hour stimulation with bemiparin and was similar to that reported in control culture - without stimulation. The excessive release of $\mathrm{TF}$ may results from the markedly stronger activation of its surface during UFH application. Hence, LMWHs administration seems to be markedly more profitable [6]. Salez et al. [7] obtained opposite results after stimulation of endothelial cells with UFH and observed no significant changes in TF concentration regardless the incubation time. However, administration of enoxaparin had similar effects to the stimulation with bemi-
Table 2. Effect of UFH vs LMWHs on anti- and procoagulant factors in HUVEC culture.

\begin{tabular}{|c|c|c|c|}
\hline & UFH & LMWH & Literature \\
\hline TF & $\uparrow$ & n.im. & 7 \\
\hline TF $(+ \text { LPS })^{1}$ & $\downarrow$ & $\downarrow \downarrow$ & 9 \\
\hline $\mathrm{TF}(\mid \mathrm{LI} S)^{\mathrm{II}}$ & $\downarrow$ & $\downarrow$ & 9 \\
\hline TF $(\mid \mathrm{LPS}){ }^{\mathrm{III}}$ & $\downarrow$ & $\downarrow$ & 9 \\
\hline $\mathrm{TF}$ & n.im. & n.im. & 8 \\
\hline TFPI & $\uparrow$ & $\uparrow \uparrow$ & $14,8,11$ \\
\hline TFPI & $\uparrow$ & $\uparrow$ & 9 \\
\hline TM & $\uparrow^{\mathrm{IV}} /$ n.im. $^{\mathrm{V}}$ & $\uparrow$ & 9 \\
\hline TM (+LPS) & $\uparrow^{\mathrm{TV}} /$ n.im. $^{\mathrm{V}}$ & $\uparrow$ & 9 \\
\hline vWF & $\downarrow$ & $\downarrow$ & 8,15 \\
\hline PAI-1 & n.im. & n.im. & 7 \\
\hline PAI-1 & $\uparrow$ & $\uparrow$ & 7 \\
\hline t-PA & $\uparrow \uparrow$ & $\uparrow$ & 7 \\
\hline
\end{tabular}

Abbreviation: I. 1-4 hour - incubation in HMEC-1, II. 1-4 hour- incubation in HUVEC, III. 24 hour- incubation in HMEC-1 and HUVEC, IV. in culture of HUVEC, V. in culture of HMEC-1, HMEC-1 (Human Microvascular Endothelial Cells), HUVEC (Human Umblical Vein Endothelial Cells), (+LPS)- after stimulation with lipopolisaccharide, TF - Tissue Factor, TFPI - Tissue Factor Pathway Inhibitor, TM - Thrombomodulin, PAI-1 - Plasminogen Activator Inhibitor-1, t-PA - Tissue-type Plasminogen Inhibitor

parin. Interestingly, heparins decrease the enhanced activity of antigen as well as mRNA TF expression after stimulation of endothelial cells with lipopolisaccharide (LPS). These tests were carried out in two different types of endothelial cell cultures: human microvascular (HMEC-1) and macrovascular (HUVEC) endothelial cells. Neutralization of the enhanced TF level by means of LPS in HMEC-1 was the most efficient in case of dalteparin. In HUVEC, dalteparin and UFH inhibited similarly TF activity, antigen as well as mRNA expression. These results refer to the short time (about 4 hours) of heparin administration, whereas, in case of longer stimulation (24 hours), the effects of TF inhibition were comparable in both cultures of HMEC-1 and HUVEC, regardless of heparin used [8].

Heparins also affect the release of TFPI - serine protease, mainly produced by cells of vascular endothelium cells, simultaneously being storage of this inhibitor. The role of TFPI is to inhibit the coagulative activity of TF/VIIa complex, as well as an inactivation of factor Xa $[9,7]$. TFPI is built up of the N-ending region formed by 3 domains: the first binding VIIa factor, the second associated with Xa factor and the third, possessing capability of heparin complexing. The Cending fragment plays an extremely significant role in the anticoagulant activity of TFPI $[10,11]$. TFPI can be 
found in a free form or a form bound with LDL, the latter predominating in the plasma. The unbound form plays the most important role in the anticoagulant activity of TFPI. It is released from the intracellular storages upon heparin's stimulation and fulfill as EC activation/injury indicator [12]. Among the others Hansen et al. performed studies to confirm TFPI increase after UFH administration [10]. TFPI concentrations in the supernatant from HUVEC culture and its amount on the surface of culture cells were compared. The significant increase of TFPI concentration was observed in the fluid fraction but was unchanged in the HUVEC surface. A hypothesis of heparin's stimulating effect on the release of intracellular TFPI was put forward [10]. The studies on the differences between the influence of UFH and LMWHs on the release of TFPI in vitro proved significant superiority of LMWH - enoxaparin [7]. The increase in the concentration of unbound/free TFPI in HUVEC culture depended on the incubation time. A significant level was obtained after 8 hours of enoxaparin application, whereas during EC stimulation with UFH the increased release of TFPI was reported after 24 hours and was lower in comparison with LMWH [7]. Westmucket's et al. studied two LMWHs: dalteparin and bemiparin in the culture and confirmed a more potent effect of LMWHs than UFH on TFPI concentration [13]. Interesting results were obtained after changing the infusion conditions (e.g. hydrostatic pressure, shear stress, flow rate) to get various physiological types of flows (venous and aortic). The results were compared to stimulation with the same heparins in static conditions. Under the conditions similar to those in the aortic flow, the TFPI intracellular secretion, its concentration on the endothelial surface and even its mRNA expression were significantly higher in case of bemiparin, whereas UFH and dalteparin had no effect on these indicators [13]. Similar results were obtained in the venous flow conditions, which may prove a better prevention against thrombin formation under bemiparin than during other heparin treatment. Based on these studies, a modulating influence of blood flow on the TFPI concentration was proved during heparin therapy. Similar tests were carried out in HMEC-1 culture. The concentration of TFPI antigen increased after UFH and LMWH (dalteparin) administration. However, no significant difference was observed between both heparins [8]. The release of lower amounts of TFPI after UFH in HUVEC culture is explained by their greater affinity to this inhibitor. This refers to both its free and bound forms $[7,8]$. Hence, LMWHs administration seems to be much more favorable, especially in case of arterial thrombosis.

Thrombomodulin, a protein found on the surface of endothelium and characterized by anticoagulat properties, belongs to the markers of EC activation. Heparins markedly change its plasma concentration, which was confirmed in HUVEC culture [8]. However, a significant increase in TM antigen, regardless the type of heparin used, being directly proportional to doses applied, was demonstrated in the study. Other results were obtained in HMEC-1 culture, where only LMWH enhanced TM concentration, whereas UFH had no influence on this parameter. Preincubation of endothelial cells in HMEC-1 with LPS gave the similar results - only LMWH modulated TM concentration [8].

The influence of heparins on fibrinolysis has not been finally explained. Few studies describing this item demonstrate an increased release of t-PA and PAI1 in HUVEC culture after heparins administration. The significantly high concentration of t-PA was obtained after stimulation of endothelial cells with UFH, whereas LMWH (bemiparin) induced its lower increase. UFH and LMWH increased PAI-1 concentration to a similar extent. To explain the mechanism responsible for PAI-1 increase, the expression of its gene was measured. However, no differences were proved between non-stimulated cells and those heparin-treated. The assumption that there was only a direct influence of heparins on endothelial cells was confirmed [6]. The studies were also carried out to discover the mechanism responsible for the release of t-PA. They confirmed its release from endothelial cell and additionally indicated Weibel-Pallade bodies as the site of t-PA reservoirs (similarly to $\mathrm{vWF}$ ) $[14,15]$.

The hypothesis about more favorable effects of LMWHs compared to UFH was put forward, based on haemostatic characteristics of both types of heparins. The unfavorable activity of UFH was mainly associated with the stronger activation of endothelium, which could be demonstrated in an enhanced TF concentration in the culture [6]. Apart from this, UFH had significantly less effect on the release of TFPI than LMWHs, thus restricting the inhibition of blood coagulation.

\section{Influence of different heparins on angiogene- sis in HUVEC culture}

Angiogenesis (forming new vessels from pre-existing structures) is a process, responsible for the development of cancer, which conditions both the growth and formation of metastases. Migrating endothelial cells and growth factors such as a vascular endothelial growth factor (VEGF) or fibroblast growth factor (FGF) play a key role in this process.

In recent years, it was demonstrated that heparins affect angiogenesis, among the others, via inhibition of growth factors. However, studies carried out in HUVEC culture proved significant differences between different heparins in this action. Cultured endothelial cells were stimulated by FGF-2 and 
VEGF165 and different heparins: UFH and LMWHs (6 kDa, $3 \mathrm{kDa}, 2.4 \mathrm{kDa}, 1.7 \mathrm{Da}, 1.2 \mathrm{kDa})$. Heparin of medium molecular weight $(6 \mathrm{kDa})$ inhibited the most effectively cell proliferation and loop formation. Unfractionated heparins and LMWH (3 kDa) inhibited angiogenesis to a lesser extent. No expected results were observed in the case of lower than $3 \mathrm{kDa}$ fractions [16]. These studies confirmed earlier findings about the modulating effect of heparan sulphate proteoglycans (HSPG), which are co-receptors responsible for dimerization of FGF and VEGF and their binding to a specific receptor on the endothelial cells (and in this way - the activation of angiogenesis). Heparins of molecular weight less than $3 \mathrm{kDa}$ were similar structurally to sulphates, and therefore they had no such effects as high-chained heparins that inhibited growth factors binding to HSPG.

Collen et al. [17] explained that a better antiangiogenic activity of LMWHs might have a different impact on the structure and mechanical properties of fibrin that constituted 'a scaffold' for the development of cancer and played a main role in the formation of capillary-like structures. LMWHs enabled the formation of a tight and fine fibrin net, whereas UFH contributed to the development of thick and porous fibers. Based on this study, the conclusion of more favorable anticancerous effects during LMWHs therapy was put forward. However, taking into consideration the fact that heparins operate similarly also when fibrin is absent in the culture, this explanation seems exceptionally inaccurate. Hence, the explanation demonstrating the influence of heparins on the receptive mechanism of growth factors activity seems to be more accurate.

Marchetti et al. studies [18] also confirmed a more profitable anticancerous effect of LMWHs in a cell culture of HMEC-1. Apart from standard using recombined VEGF165 and FGF-2, they also applied agents produced by two different types of neoplastic cell lines of breast cancer and leukemia. They were the first to observe not only heparin-induced inhibition of endothelium proliferation but also the formation of vascular capillaries caused by these medications. The studies confirmed explicitly that LMWHs inhibited markedly better both proliferation and formation of new vascular loops regardless the type of growth factors used. The anticancerous activity of LMWHs e.g., dalteparin and enoxaparin was compared. Enoxaparin appeared to be especially active in the inhibition of angionenesis induced by growth factors [18]. Additionally, a main role of VEGF in formation of vessels was proved. Its concentration was higher in neoplastic cell culture compared to other released cytokines (FGF-2, TNF- $\alpha$ ) [18]. A modulating effect of heparins on differentiation and reorganization of cells induced by an agent coming from neoplasm culture was demonstrated in these studies.
The mechanism of heparins anticancerous effect connected with TFPI release from the endothelium stimulated by LMWH (tinzaparin) and UFH was presented [19]. It was proved that TF/VIIa complex, similarly to FGF-2, activated the formation of new vessels. To confirm the role of TFPI in angiogenesis inhibition, LMWH or a recombined TF inhibitor (rTFPI) was added to HUVEC culture stimulated by FGF-2 or TF/VII. The results proved that both LMWH and rTFPI block the induction of angiogenesis, caused by both activators. Monoclonal antibodies of TFPI, applied in the culture, eliminated an early antiangiogenic effect, which confirmed explicitly the role of TFPI in inactivation of this process. Tinzaparin was more efficient than UFH in the inhibition of new vessels formation, which is associated with higher release of TFPI.

Summing up, low molecular weight heparins, regardless the anticancerous mechanism (receptor type or associated with TFPI release), showed the higher activity than UFH. Based on the assumption that cancer requires higher amounts of glucose to its growth, and its cells are characterized by an increased expression of glucose transporters (GLUTs), a hypothesis about the possibility of inhibiting these processes by glucosylated heparins $(\mathrm{GH})$ was put forward [20]. The mechanism of anticancerous activity of these compounds consists in blocking GLUTs. Modified heparins unable tumor's cells to absorb glucose and cause their necrosis. It was established simultaneously that the fewer radicals of glucosamine in the structure of $\mathrm{GH}$, the more potent effect of proliferation inhibition in HUVEC.

Reversely, the anti-Xa activity of these compounds intensifies itself together with the increased number of glucosamine radicals, which may decrease the risk of hemorrhages after administrating more GH. Apart from this, GH showed no toxic effects on endothelial cells coming from HUVEC culture [20].

\section{Influence of UFH vs LMWHs on OPG/RANKL/RANK system}

In recent years the interest of many researchers has been focused on the influence of heparins on the induction of osteoporosis and initiation of vessels' calcification connected with atherosclerosis. These both mechanisms are related to osteoprotegerin (OPG), which is a soluble receptor for a RANK ligand receptor (RANKL; Receptor Activator of Nuclear Factor NF- $\kappa B$ Ligand). The role of OPG is to block the activation of RANK via binding it to its ligand - RANKL that inhibits osteoclastogenesis - a loss of bone mass [21].

Heparins, small doses of UFH and big doses of LMWHs, cause the release of similar amounts of OPG 
from endothelium in HUVEC culture [22]. Additionally, they are able to bind OPG. Especially UFH strongly influences the activation of osteogenesis [23]. C-terminal end region of OPG is responsible for heparins binding. This discovery has brought us closer to understanding the whole mechanism responsible for intensification of osteoporosis during long-term treatment with heparins [22]. The examinations performed so far focused mainly on the role of heparins as activators of osteoporosis. However, another unexplained problem is the influence of these drugs on atherogenesis and vessel calcification via OPG/RANKL/RANK system. So far, only one finding showing a correlation between OPG and vWF has been observed [4]. Osteoprotegerin is physiologically connected with $\mathrm{VWF}$, being at the same time a well known marker of vessel damage by means of A1 domain present in its molecule. These studies proved only the intracellular formation of vWF-OPG complex. This interaction requires a high concentration of calcium ions and a low level of chloride ions and optimum $6.5 \mathrm{pH}$. Since these conditions are intracellular, the hypothesis of absence of vWFOPG complex in the blood vessels has been presented. However, up to date, there is no explicit proof confirming this thesis. OPG and $\mathrm{vWF}$ are probably formed in the net of the Golgy apparatus and stored as complexes in the Weibel-Pallade bodies [4]. The enhanced vWF concentration correlated with the extent of damage to endothelium and was one of the thrombosis risk factors [24]. Despite the certain fact that heparins cause the activation of endothelial cells, the increased release of vWF due to their activity has not been proved. Interestingly, the studies carried out in HUVEC have shown a decrease in VWF concentration regardless the type of heparin used [8]. The influence of heparins on vWF levels in vitro has not been explained. There are no findings confirming and explaining the effect of OPG/RANKL/RANK complex on the development of vessel calcification and its relation to heparins therapy. Further studies in HUVEC may help to understand this problem.

\section{Summary and conclusion}

UFH and LMWHs differ significantly in pharmcokinetics, bioaccessibility and in numerous other properties of great clinical significance. Low-molecular weight heparins activate endothelial cells to a lesser extent than UFH. However, they influence more potently to TFPI release than unfractionated heparins. The profit of LMWHs therapy is also observed in case of anticancerous mechanisms of heparins. The inhibition of angiogenesis induced by growth factors and TF/VIIa complex is better under LMWHs application. Summing up, LMWHs predominate over UFH, both with regard to haemostatic and anticancerous properties, as well as side effects of their administration. Another interesting problem is the influence of various types of heparins on the OPG/RANKL/RANK system. It is associated with two different processes: intensified osteoporosis and heparin effect on vessel calcification associated with atherosclerosis. Despite many years of studies, pleiotropic properties of heparins have not been fully explained and are still in the focus of attention to many researchers. Studies provided with human vessel cultures may have special significance for the explanation of heparins' effect on endothelium.

\section{References}

[1] Chojnowski K. Miejsce i rola heparyn drobnocząsteczkowych $\mathrm{w}$ profilaktyce i leczeniu procesów zakrzepowo-zatorowych - 20 lat doświadczeń klinicznych. Nowa Klin. 2000;7(6):661-5.

[2] Dąbrowska J. Biologiczne i terapeutyczne właściwości heparyny. Med Wet. 1996;52(11):692-6.

[3] Drożdż M, Kowalczyk-Michałek M, Kraśniak A, Sułowicz W. Współczesne poglądy na temat zastosowania heparyn drobnoczasteczkowych u chorych dializowanych. Przegl Lek. 2005;62(4):257-9.

[4] Shahazi S, Lenting PJ, Fribourg C, Terraube V, Denis CV, Christophe OD. Characteristic of the interaction between von Willebrand factor and osteoprotegerin. J Thromb Haemost. 2007;5:1956-62.

[5] Wnuczko K, Szczepański M. Śródbłonek - charakterystyka i funkcje. Pol Merk Lek. 2007;23:60-5.

[6] Pérez-Ruiz A, Montes R, Carrasco P, Rocha E. Effects of low molecular weight heparin, bemiparin, and unfractionated heparin on haemostatic properties of endothelium. Clin Appl Thromb Hemost. 2002;8(1):65-71.

[ 7] Martinez-Salez V, Vila V, Réganon E, Garcia Oms J, Aznar J. Effect of unfractionated heparin and a low molecular weight heparin (enoxaparin) on coagulant activity of cultured human endothelial cells. Haematologica. 2003;88:694-9.

[ 8] Vignoli A, Marchetti M, Balducci D, Falanga A. Differential effect of low-molecular weight heparin, dalteparin, and unfractionated heparin on microvascular endothelial cell haemastatic properties. Haematologica. 2006;91:207-14.

[9] Hansen JB, Olsen R, Webster P. Association of tissue factor pathway inhibitor with human umbilical vein endothelial cell. Blood. 1997;9(1):3568-78.

[10] Hansen JB, Svensson B, Olsen R, Ezban M, Osterud B, Paulssen RH. Heparin induces synthesis of tissue factor pathway inhibitor from endothelial cells in vitro. Thromb Heamost. 2000;83:937-43.

[11] Kato H. Regulation of functions of vascular wall cells by tissue factor pathway inhibitor. Arterioscler Thromb Vasc Biol. 2002;22:539-48.

[12] Kawaguchi A, Miyao Y, Noguchi T et al. Intravascular free tissue factor pathway inhibitor is inversely correlated with HDL cholesterol and postheparin lipoprotein lipase but proportional to Apolipoprotein A-II. Arterioscler Thromb Vasc Biol. 2000;20:251-8.

[13] Westmuckett AD, Kakkar VV, Hamuro T, Lupu F, Lupu C. Bemiparin and fluid flow modulate the expression, activity and release of tissue factor pathway inhibitor in human endothelial cells in vitro. Thromb Haemost. 2001;86:1547-54.

[14] Rosnoblet C, Vischer UM, Gerard RD, Irminger JC, Halban PA, Kruithof EKO. Storage of tissue - type plasminogen activator in Weibell-Pallade bodies of human endothelial cells. Arterioscler Thromb Vasc Biol. 1999;19:1796-802. 
[15] Huber D, Cromer EM, Kaufmann JE, Meda P, Massé JM, Kruithof EKO. Tissue - type plasminogen activator (t-PA) is stored in Weibel-Pallade bodies in human endothelial cells both in vivo and in vitro. Blood. 2002;99(10):3637-45.

[16] Koharna AA, Sahni A, Altland OD, Francis CW. Heparin inhibition of endothelial cell proliferation and organization is dependent on molecular weight. Arterioscler Thromb Vasc Biol. 2003;23:2110-15.

[17] Collen A, Smorenburg SM, Peters E et al. Unfractionated and low molecular weight heparin affect fibrin structure and angiogenesis in vitro. Cancer Res. 2000;60:6196-200.

[18] Marchetti M, Vignoli A, Russo L, Balducci D et al. Endothelial capillary tube formation and cell proliferation induced by tumor cells and affected by low molecular weight heparins and unfractionated heparin. Thromb Res. 2008;121:637-45.

[19] Mousa SA, Mohamed S. Inhibition of endothelial cell tube formation by the low molecular weight heparin, tinzaparin, is mediated by tissue factor pathway inhibitor. Thromb Haemost. 2004;92:627-33.
[20] Lee GY, Kim SK, Byun Y. Glucosylated heparin derivatives as non-toxic and anticancer drugs. $J$ Control Release. 2007; 123:46-55.

[21] Boyce BF, Xing L. Biology of RANK, RANKL, and osteoprotegerin. Artritis Res Ter. 2007;9(Suppl.1):S1.

[22] Vik A, Brodin E, Sveinbjornsson B, Hansen JB. Heparin induces mobilization of osteoprotegerin into the circulation. Thromb Haemost. 2007;98:148-54.

[23] Irie A, Takami M, Kubo H, Sekino-Suzuki N, Kasahara K, Sanai Y. Heparin enhances osteoclastic bone resorption by inhibiting osteoprotegerin activity. Bone. 2007;41:165-74.

[24] Becker CR. The investigation of biomarkers in cardiovascular diseases: time for a coordinated, international effort. European Heart Journal. 2005;26(5):421-2.

Submitted:17 November, 2008 Accepted after reviews: 5 January, 2009 